\title{
Propuesta de optimización de metas del área de mantenimiento mediante programación lineal por metas
}

\section{Optimization proposal for maintenance area goals through goal programming}

HERRERA-SÁNCHEZ, Gustavo†*, SILVA-JUÁREZ, Alejandro, GALLARDO-NAVARRO, José Luis y RÍOS-REYES, Josué Horacio

Universidad Tecnológica de Puebla, División de Mantenimiento Industrial, Antiguo Camino a La Resurrección 1002 - A, Zona Industrial, C.P. 72300 Puebla, Pue.

ID $1^{\text {er }}$ Autor: Gustavo, Herrera-Sánchez / ORC ID: 0000-0001-5276-5062, ID Thomson: F-6595-2018, arXiv Author ID: herreragh, CVU CONACYT ID: 459805

ID $1^{\text {er }}$ Coautor: Alejandro, Silva-Juárez / ORC ID: 0000-001-8473-9803, ID Thomson: F-6969-2018, arXiv Author ID: alejandrosilva1, CVU CONACYT ID: 637028

ID $2^{\text {do }}$ Coautor: José Luis, Gallardo-Navarro / ORC ID: 0000-0001-7954-4905, ID Thomson: F-8798-2018, arXiv Author ID: GALLARDO\#1, CVU CONACYT ID: 629796

ID $3^{\text {er }}$ Coautor: Josué Horacio, Ríos-Reyes / ORC ID: 0000-0001-5432-384X, arXiv Author ID: 3052294

DOI: $10.35429 /$ JCA.2020.14.4.1.7

Recibido Marzo 25, 2020; Aceptado Junio 29, 2020

Resumen

En cualquier organización todos sus procesos están interrelacionados para lograr los objetivos estratégicos de la misma. El propósito de este estudio es proponer un modelo de programación lineal por metas para la optimización de los objetivos del área de mantenimiento. La programación lineal es una técnica matemática que permite la optimización de los procesos analizando las variables que intervienen en éstos mediante algoritmos matemáticos. La primera etapa es la formulación del modelo matemático caracterizado por los indicadores del área de mantenimiento y los objetivos estratégicos, la segunda etapa es la solución del modelo matemático de programación lineal por metas considerando diferentes escenarios. La última etapa es la validación por expertos del área de mantenimiento antes de implementarla en las organizaciones. El beneficio esperado es que sea una herramienta para la toma de decisiones en el área de mantenimiento para considerar que sus actividades se pueden optimizar ya sea para aumentar sus beneficios y/o disminuir sus costos mediante la técnica de programación lineal por metas.

Programación lineal por metas, Optimización, Mantenimiento

\begin{abstract}
In any organization all its processes are interrelated to achieve its strategic objectives. The purpose of this study is to propose a goal programming model for the optimization of the objectives of the maintenance area. Linear programming is a mathematical technique that allows the optimization of processes by analysing the variables involved in them through mathematical algorithms. The first stage is the formulation of the mathematical model characterized by the indicators of the maintenance area and the strategic objectives, the second stage is the solution of the mathematical model of goal programming considering different scenarios. The last stage is the validation by experts of the maintenance area before implementing it in the organizations. The expected benefit is that it is a tool for decision making in the maintenance area to consider that your activities can be optimized either to increase your benefits and / or decrease your costs through the technique of goal programming.
\end{abstract}

Goal programming, Optimization, Maintenance

Citación: HERRERA-SÁNCHEZ, Gustavo, SILVA-JUÁREZ, Alejandro, GALLARDO-NAVARRO, José Luis y RÍOSREYES, Josué Horacio. Propuesta de optimización de metas del área de mantenimiento mediante programación lineal por metas. Revista de Cómputo Aplicado. 2020. 4-14: 1-7.

\footnotetext{
* Correspondencia del Autor (Correo Electrónico: herreragh@yahoo.com.mx)

$\uparrow$ Investigador contribuyendo como primer autor.
} 


\section{Introducción}

En las empresas se tiene como objetivo principal maximizar los beneficios y minimizar los costos, para ello se utilizan diferentes métodos y herramientas. Después de haber definido los objetivos alineados a la filosofía de la empresa, se desarrollan las estrategias para lograrlos para evaluar las actividades realizadas. Una de las tareas de un gerente es evaluar lo qué y cómo se hace, ya que la utilización de los recursos debe ser racional y aplicarlos dónde se requieran (Drucker, 2020).

Por ejemplo, para el área de producción Lean manufacturing con sus dos enfoques de minimizar los desperdicios, que se traducen en costos, y maximizar el valor agregado a los productos (Castillo Flores, Arellano Briones, \& Fernández García, 2018). En Logística, sistematizar la planeación de la logística con la finalidad de obtener mayor eficiencia y eficacia en su operación mediante la Teoría de Sistemas (Arrellano González, Carballo Mendivil, Acosta Quintana, \& López Torres, 2017).

Por otra parte, que efectos provocan los recursos de mantenimiento en los niveles de proyectos seis sigma en el proceso de mejora continua (Chávez Medina, Santiesteban López, Carmona Silva, \& Muñiz Montero, 2018). También, como el Mantenimiento Productivo Total (TPM) aumenta la productividad y la reducción de productos no conformes (Moreno Vázquez \& Castillo Valdez, 2018).

La Programación Lineal (PL) es una técnica matemática basada en modelos matemáticos y su solución mediante algoritmos matemáticos como el método Simplex desarrollado por Robert B. Dantzig (Albers, Reid, \& Dantzig, 1986), considerado el padre de la PL. Existen diferentes fenómenos físicos, químicos, de negocios, de economía, entre otros, que pueden resolverse mediante PL dependiendo de sus características. Así tenemos diferentes métodos de PL como programación lineal continua, programación lineal entera o discreta, problemas de redes (Martínez S., Vertiz C., López P., Jiménez L., \& Moncayo M., 2014), problemas de transporte, problemas de transbordo, programación por metas u objetivos, programación dinámica.
Estos modelos de PL se caracterizan por su naturaleza lineal, por lo que se considera una herramienta ampliamente utilizada y efectiva para la resolución de problemas en los que se desea obtener mayor provecho de alguna situación.

La contribución de este estudio es proponer un modelo de programación por metas para el área de mantenimiento industrial para el uso racional de los recursos utilizados en sus actividades y sirva como un elemento para la toma de decisiones.

\section{Evaluación del Mantenimiento Industrial}

El área de mantenimiento industrial en las organizaciones tiene igual importancia que las demás áreas, pues de acuerdo con la teoría de sistemas existe una interrelación entre las áreas de las empresas y todas están enfocadas a un objetivo común, generar beneficios económicos y reducir costos. Este objetivo común tiene que ser optimizado, maximizando los beneficios y minimizando los costos, por todo el sistema y sus subsistemas. En primera instancia para establecer las metas u objetivos se requiere tener como base la definición del concepto de mantenimiento industrial. De acuerdo con la norma británica BS 3811 es "La combinación de todas las acciones técnicas y administrativas asociadas tendientes a conservar un ítem o restablecerlo a un estado tal que pueda realizar la función requerida". Indica, además que la función requerida puede ser definida como una condición dada.

Como todo sistema, el área de mantenimiento industrial debe tener un objetivo alineado al objetivo general de la empresa. Hay diferentes enfoques para evaluar el funcionamiento del área de mantenimiento: por medio del enfoque sistémico e integral confiabilidad, mantenibilidad y confiabilidad (Mora G., 2013); el enfoque de mantenimiento total de la producción mediante el indicador Eficiencia Global del Equipo (EGE) que involucra las seis grandes pérdidas en un proceso (Rey S., 2003); por medio de índices de eficiencia y efectividad, costo de mantenimiento (Louit, 2012); el enfoque de mantenimiento centrado en la confiabilidad (Moubray, 2004); utilizando un benchmarking como elemento de comparación para el funcionamiento de mantenimiento (Castillo F., Estrada C., Pérez O., \& Ríos R., 2017), entre otros enfoques. 


\section{Marco Teórico de la Programación Lineal}

Desde que George B. Dantzig en 1947, presento un problema de programación lineal hasta la actualidad, esta técnica matemática es utilizada para optimizar los procesos y sus recursos en muchas empresas. La PL se clasifica como PL con variables continuas, variables discretas, binarias y por metas (objetivos), además que, otros eventos se pueden formular como problemas de PL, por ejemplo, programación dinámica, redes, modelos de transporte, modelos de asignación (Taha, 2012), entre otros.

Las condiciones principales de la PL es su linealidad, determinismo, en general un solo objetivo, la función objetivo, y la satisfacción de las restricciones. El modelo matemático general se presenta en la ecuación (1).

Máx o Mín $Z=\sum_{j=1}^{n} c_{j} x_{j}$

Sujeto a:

$\sum_{\substack{j=1 \\ x_{j} \geq 0}}^{n} a_{j} x_{j}(\leq,=, \geq) b_{j}$

Sin embargo, no todos los problemas de PL tienen un objetivo o función objetivo. Existen otros modelos que tienen en consideración metas individuales o múltiples porque hay metas antagónicas, contrarias o de diferentes dimensiones.

La programación por metas estudia problemas lineales que tengan objetivos múltiples y cuantificables (Budnick, 2007). Además, se requiere una clasificación ordinal por rangos para establecer metas primarias y secundarias. Las metas de primer orden serán resueltas inicialmente, ya que éstas son la primera prioridad para el gerente de mantenimiento puesto que contribuyen a la eficacia (externas al área de mantenimiento) se consideran esenciales para la contribución al objetivo general. Después las de segundo orden que pueden ser propias de la eficiencia (el uso racional de los recursos asignados al área de mantenimiento). El modelo general de programación por metas es (Álvarez, 2019), dados un conjunto de soluciones, $\mathrm{S} \subseteq \mathrm{R}^{\mathrm{N}}, \mathrm{S} \neq$ $\emptyset, f_{j}: S \rightarrow R, j \in\{1, \cdots, p\}$, el problema de optimización multiobjetivo se plantea en los términos: $\min \left(f_{1}(x), \ldots, f_{p}\right)$

s. a.: $x \in S$

tiene una solución óptima en $\bar{x}^{j}$ con valor en $f_{j}^{*}$.

Para un problema de programación por metas, el primer paso es fijar los objetivos $f(x)$, que se consideren relevantes. El segundo paso es determinar el nivel de logro, $t$, que corresponda a cada objetivo. Cada meta se convierte en una restricción para incorporarla en el modelo mediante las siguientes variables de desviación:

- $d_{i}^{+}$: variable de desviación positiva, cuantifica el exceso de logro de una meta.

- $d_{i}^{-}$: variable de desviación negativa, cuantifica la falta de logro de una meta.

Introduciendo las variables de desviación y un vector de logro $a=\left(a_{1}, a_{2}, \ldots, a_{p}\right)$, donde $a_{i}=g_{i}\left(d_{i}^{+}, d_{i}^{-}\right), \quad i=1,2, \ldots, p$ en el modelo matemático de programación por metas (ecuación 3):

mín $\left(a_{1}, \ldots, a_{p}\right)$

s. $a .: f_{j}(x)+d_{j}^{-}-d_{j}^{+}=m$

$x \in S$

$d_{j}^{-}, d_{j}^{+} \geq 0, j=1,2, \ldots, p$

Ya planteado el problema de programación por metas, el siguiente paso es resolverlo mediante cualquiera de los siguientes métodos: metas lexicográficas (García, 1998) (Romero, 1993), metas ponderadas (Ishizaka \& Nemery, 2013) y método MINIMAX (Jiménez L., Rivas P, \& Zubia Z, 2008). El método seleccionado depende del objetivo planteado por el área de mantenimiento para lograr sus metas.

\section{Propuesta de programación por metas para el área de mantenimiento industrial}

La propuesta de un modelo para el área de mantenimiento inicia con la definición de las variables de decisión, $x_{j}$, las cuales son:

- $x_{1}=$ número de rutinas de mantenimiento correctivo en un período. Estás se pueden definir de acuerdo con datos históricos.

- $x_{2}=$ número de rutinas de mantenimiento preventivo en un período.

HERRERA-SÁNCHEZ, Gustavo, SILVA-JUÁREZ, Alejandro, GALLARDO-NAVARRO, José Luis y RÍOS-REYES, Josué Horacio. Propuesta de optimización de metas del área de mantenimiento mediante programación lineal por metas. Revista de Cómputo Aplicado. 2020 
- $\quad x_{3}=$ número de rutinas de mantenimiento predictivo en un período.

$\mathrm{Si}$ suponemos que en una empresa industrial tenemos una serie de máquinas críticas en la línea de producción o varias líneas de producción para la fabricación de los productos y de acuerdo con un Plan Maestro de Mantenimiento se tendrá la planificación y programación de las rutinas de mantenimiento preventivo y predictivo con sus tiempos requeridos, sus costos asociados y la mano de obra requerida (Tavares).

Derivado del Plan Maestro de Mantenimiento se obtiene el presupuesto en un período determinado para el área de mantenimiento incluyendo las refacciones y suministros, mano de obra directa e indirecta, maquinaria y equipo a utilizar.

Por lo tanto, se establecen las restricciones para las líneas de producción en relación con el tiempo programado para las rutinas de mantenimiento y del costo (ecuación 4).

$$
\begin{aligned}
& a_{11} x_{1}+a_{12} x_{2}+\cdots+a_{1 i} x_{j} \leq b_{1} \\
& a_{21} x_{1}+a_{22} x_{2}+\cdots+a_{2 i} x_{j} \leq b_{2} \\
& \vdots \quad \vdots \quad \quad \vdots \quad \leq \quad \vdots \\
& a_{j 1} x_{1}+a_{j 2} x_{2}+\cdots+a_{j i} x_{j} \leq b_{j}
\end{aligned}
$$

Por ejemplo, si suponemos que se tiene la siguiente información de una línea de producción con sus costos promedio para una línea de producción en cierto período $\$ 3,000$ para la rutina de mantenimiento correctivo, $\$ 2,500$ para la rutina de mantenimiento preventivo y $\$ 4,500$ para la rutina de mantenimiento predictivo. En lo referente al tiempo promedio de las rutinas de mantenimiento correctivo, preventivo y predictivo para cierto período estos son 3.0 horas, 3.5 horas y 4.0 horas, respectivamente.

De acuerdo con el historial de las órdenes de trabajo se tiene en promedio 4 para mantenimiento correctivo, y como referencia del Plan Maestro de Mantenimiento en promedio es 10 órdenes de mantenimiento preventivos y 2 órdenes de mantenimiento predictivo.
De esta información se generan las políticas de mantenimiento, como máximo 4 rutinas de mantenimiento correctivo, 2 rutinas de mantenimiento predictivo y en especial el cumplimiento de las 10 órdenes para el mantenimiento preventivo con la finalidad de coadyuvar a lograr el plan de producción en ese mismo período.

Se considera adecuado el $20 \%$ para el mantenimiento correctivo y el $80 \%$ para el mantenimiento preventivo del presupuesto total de mantenimiento (Sadi, 2014). Para esta propuesta se considera el $20 \%, 60 \%$ y $20 \%$ del presupuesto total para el mantenimiento correctivo, preventivo y predictivo, respectivamente. Los recursos disponibles son: $\$ 50,000$ como presupuesto de los costos variables de mantenimiento; 70 horas de mano de obra directa.

Con esta información se genera el modelo de programación lineal:

$\operatorname{Min} Z=3000 x_{1}+2500 x_{2}+4500 x_{3}$

Sujeto a:

$$
\begin{aligned}
& 3 x_{1}+3.5 x_{2}+4 x_{3} \leq 70 \\
& x_{1} \leq 4 \\
& x_{2} \quad \geq 10 \\
& x_{3}=2 \\
& 3000 x_{1} \leq 50000(0.2) \\
& 2500 x_{2} \leq 50000(0.6) \\
& 4500 x_{3} \leq 50000(0.2) \\
& x_{i} \geq 0 \text { y enteros }
\end{aligned}
$$

$\mathrm{El}$ área de mantenimiento puede requerir metas múltiples, no solo, lograr un costo mínimo del mantenimiento. Se consideran prioridades para este logro de metas. La primera meta es lograr el presupuesto asignado, la segunda meta es el cumplimiento del tiempo disponible y la tercera meta es que se cumplan las rutinas de mantenimiento preventivo de acuerdo con el Plan Maestro de Mantenimiento.

A continuación, se enuncian las variables de desviación para las metas:

$d_{1}^{+}=$Variable de desviación no deseada para el presupuesto asignado para mantenimiento. 
$d_{1}^{-}=$Variable de desviación deseada para el presupuesto asignado para mantenimiento.

$d_{2}^{+}=$Variable de desviación no deseada para el tiempo promedio requerido para las rutinas de mantenimiento.

$d_{2}^{-}=$Variable de desviación deseada para el tiempo promedio requerido para las rutinas de mantenimiento.

$d_{3}^{+}=$Variable de desviación no deseada para el número de rutinas de mantenimiento preventivo.

$d_{3}^{-}=$Variable de desviación deseada para el número de rutinas de mantenimiento preventivo.

La función objetivo de la programación por metas ponderadas es:

$\operatorname{Min} Z=1 d_{1}^{+}+0.5 d_{2}^{+}+0.25 d_{3}^{+}+0.25 d_{3}^{-}$

Se establecen las metas:

$$
\begin{array}{rrr}
3000 x_{1}+2500 x_{2}+4500 x_{3}+d_{1}^{-}-d_{1}^{+} & =50000 \\
3 x_{1}+3.5 x_{2}+ & 4 x_{3}+d_{2}^{-}-d_{2}^{+} & =70 \\
x_{2}+ & d_{3}^{-}-d_{3}^{+} & =10
\end{array}
$$

Las restricciones estructurales son:

$x_{1} \leq 4$

$x_{3}=2$

$3000 x_{1} \leq 10000$

$2500 x_{2} \leq 30000$

$4500 x_{3} \leq 10000$

La condición de no negatividad es:

$x_{i} \geq 0, d_{i}^{+} \geq 0, d_{i}^{-} \geq 0$ y enteros

En la figura 1, se muestra parte de la solución del modelo de programación por metas ponderadas realizada por medio de Solver Excel.

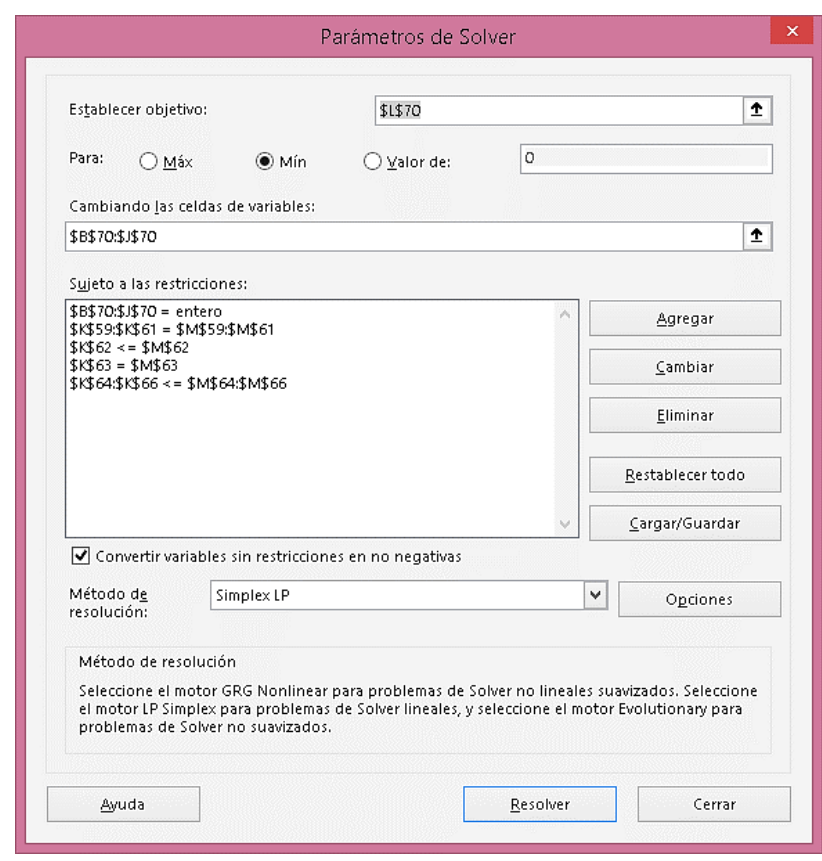

Figura 1 Solución del modelo por medio de Solver Excel

Fuente: Solver-Excel

La solución indica que el valor de $\mathrm{Z}=0$, lo cual indica que las 3 metas se han logrado, pues los valores de la función objetivo son cero, decir, $d_{1}^{+}=0, d_{2}^{+}=0, d_{3}^{+}=0$ y $d_{3}^{-}=0$. Ver figura 2 .

La solución para las variables de decisión es: $x_{1}$, el número de rutinas de mantenimiento correctivo son 3 de las 4 planificadas, $x_{2}$, las rutinas de mantenimiento preventivo son $10 \mathrm{de}$ acuerdo con la meta ponderada establecida como una igualdad $\mathrm{y}$, por último, $x_{3}$, las rutinas de mantenimiento predictivo son 2 .

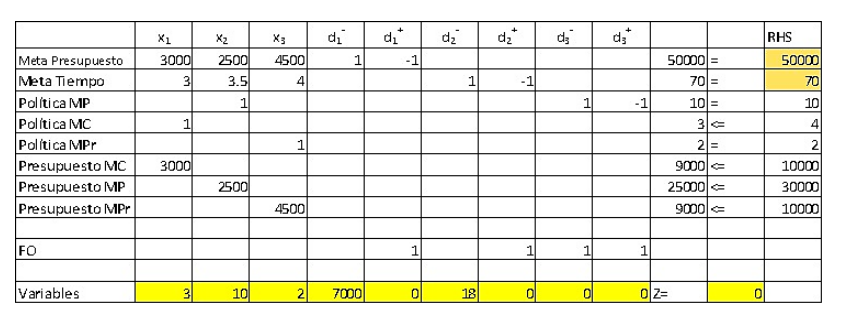

Figura 2 Solución del problema de metas con Solver Fuente: elaboración propia

La variable de desviación $d_{1}^{-}=7000$ indica que hay un sobrante del presupuesto total de mantenimiento. La variable $d_{2}^{-}=18$ muestra que las horas de mano de obra directa tiene un sobrante de 18 horas. Con esta información se puede crear otro escenario de reducir el presupuesto y reducir el tiempo de mano de obra disponible de acuerdo con los resultados de las variables de desviación y ver si sigue vigente el logro de las tres metas deseadas. 
Como consecuencia del diseño del modelo matemático de programación de metas, la restricción que sería disminuida es el número de rutinas de mantenimiento correctivo, ver figura 3 .

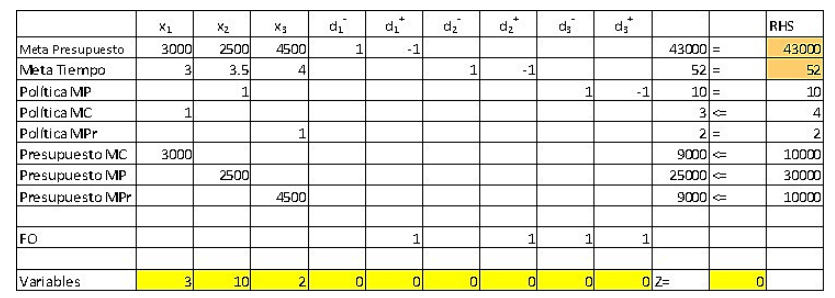

Figura 3 Cambio de escenario con los resultados de las variables de desviación

Fuente: elaboración propia

\section{Discusión}

La programación por metas nos proporciona una herramienta para la toma de decisiones en el área de mantenimiento para múltiples metas. Con sus diferentes formas de plantear la programación por metas se pueden crear diferentes escenarios para las diferentes metas que se quieran lograr.

Por ejemplo, se quiere alcanzar las metas de las rutinas de mantenimiento correctivo y preventivo. Si se tienen más de una línea de producción o más de una máquina crítica, las variables de decisión serían $x_{i j}$ que indican las $i$ - ésima rutina de mantenimiento con la $j-$ ésima máquina. Entonces, las variables de desviación para las diferentes máquinas o líneas de producción son $d_{i j}^{+}$y $d_{i j}^{-}$.

También, se pueden incorporar otros indicadores de mantenimiento como Tiempo Medio Entre Fallas, Tiempo Medio Para Reparar y Tiempo Medio Para Fallar. Así como las seis grandes pérdidas de producción analizadas por el TPM.

Por último, para disminuir la desventaja de que es un modelo determinístico, se utiliza el análisis de sensibilidad (Anderson, Sweeney, Williams, \& Camm, 2011) para analizar los cambios en los coeficientes de las metas y restricciones que afectan a la solución óptima. Ver figura 4.

\begin{tabular}{|c|c|c|c|c|c|c|}
\hline Celda & Nombre & $\begin{array}{l}\text { Final } \\
\text { Valor }\end{array}$ & $\begin{array}{c}\text { Sombra } \\
\text { Precio }\end{array}$ & $\begin{array}{l}\text { Restricción } \\
\text { Lado derecho }\end{array}$ & $\begin{array}{l}\text { Permisible } \\
\text { Aumentar }\end{array}$ & $\begin{array}{l}\text { Permisible } \\
\text { Reducir }\end{array}$ \\
\hline$\$ K \$ \$ 59$ & Meta Presupuesto RHS & 50000 & t & 50000 & $1 \mathrm{E}+30$ & 6000 \\
\hline$\$ K \$ 60$ & Meta Tiempo RHS & 70 & t & 70 & $1 E+30$ & 17 \\
\hline$\$ K \$ 61$ & Politica MP RHS & 10 & t & 10 & 2 & 10 \\
\hline$\$ K \$ 62$ & Politica MC RHS & 3.333333333 & c & 4 & $1 E+30$ & 0.666666667 \\
\hline$\$ K \$ 63$ & Politica MPr RHS & 2 & c & 2 & 0.2222222222 & 2 \\
\hline$\$ K \$ 64$ & Presupuesto MC RHS & 10000 & 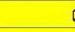 & 10000 & 2000 & 10000 \\
\hline$\$ K \$ \$ 65$ & Presupuesto MP RHS & 25000 & t & 30000 & $1 E+30$ & 5000 \\
\hline$\$ K \$ 66$ & Presupuesto MPr RHS & 9000 & c & 10000 & $1 E+30$ & 1000 \\
\hline
\end{tabular}

Figura 4 Análisis de sensibilidad con Solver Fuente: Solver-Excel

Observamos que la columna de precio sombra, todos los valores para las restricciones son cero, lo cual indica que no es necesario aumentar los recursos del lado derecho. Por ejemplo, la meta de presupuesto tiene un sobrante de dinero, por lo que no es necesario aumentar el presupuesto.

\section{Conclusión}

Las decisiones en las organizaciones siempre son relevantes para el logro de los objetivos. Es cierto que, este modelo matemático es determinístico y lineal, sin embargo, presenta la ventaja del análisis post óptimo, el cual permite generar diferentes escenarios para responder las preguntas de "qué pasa sí...", ya que, los procesos de las empresas son dinámicos, como también el entorno donde interactúa.

También permite escalar a más variables de decisión, $x_{i}$ y variables de desviación con sus respectivas nuevas metas. Esto permite la creación de diferentes alternativas que la empresa puede considerar para alcanzar sus objetivos. Por ejemplo, se puede incluir la programación por metas para mantenimiento al modelo matemático de un plan de producción con variables de decisión que sean los productos principales que aporten el mayor margen de utilidad, considerando los inventarios iniciales y finales, el costo de producción, el costo de almacenamiento, el tiempo disponible para su fabricación, entre otros factores.

Finalmente, con el análisis de sensibilidad acerca de los coeficientes de las restricciones y metas, se tienen más caminos de acción para explorar otros contextos que pueden convenir a las organizaciones 


\section{Referencias}

Albers, D., Reid, C., \& Dantzig, R. (1986). An Interview with George B. Dantzig: The Father of Linear Programming. The College Mathematics Journal, Vol. 17, No. 4. Mathematical Association of America, 292-314.

Álvarez, M. J. (2019). Programación por metas: Tesis de grado. Tenerife, España: Universidad de La Laguna.

Anderson, D., Sweeney, D., Williams, T., \& Camm, J. (2011). Métodos cuantitativos para los negocios. México: CENGAGE Learning.

Arrellano González, A., Carballo Mendivil, B., Acosta Quintana, M. P., \& López Torres, V. G. (2017). Planeación de la logística de la recepción y entrega de mercancía en una empresa que ofrece servicios de paquetería y carga. Revista de Negocios \& PyMES Junio 2017 Vol.3 No 8, 922.

Budnick, F. S. (2007). Matemáticas aplicadas para Administración, Economía y Ciencias Sociles. México: Mc Graw Hill.

Castillo F., A. L., Estrada C., F. J., Pérez O., K., \& Ríos R., V. M. (2017). Benchmarking de manteniendo industrial. Revista de Tecnología e Innovación. Vol.4 No.13 , 1 - 12 .

Castillo Flores, A. L., Arellano Briones, O. D., \& Fernández García, L. G. (2018). ¿Cuáles son las herramientas de Lean Manufacturing más utilizadadas en las empresas petroquímicas de la Zona sur de Tamaulipas? Revista de Ingeniería Industrial Septiembre 2018 Vol.2 No.5, 24-30.

Chávez Medina, J., Santiesteban López, N. A., Carmona Silva, J. L., \& Muñiz Montero, I. (2018). Efecto del mantenimiento industrial, maquinaria y equipo, mano de obra, métodos de trabajo y materia prima con respecto al nivel de Six Sigma en una Pyme: Caso Bloquera Medina del municipio de San Pedro Cholula, Puebla. Revista de Ingeniería Industrial Diciembre 2018 Vol.2 No.6, 34-44.

Drucker, P. (15 de enero de 2020). Alta Dirección. Obtenido de La innovación: http://altadireccion.com.ar/la-innovacion-porpeter-drucker/
García, A. (1998). Programación estocástica por metas. Teoría y aplicaciones económicas. Tesis Doctoral. Madrid: Universidad Complutense.

Ishizaka, A., \& Nemery, P. (2013). Multi criteria decision analysis. Wiley.

Jiménez L., M., Rivas P, J., \& Zubia Z, M. (2008). Un modelo de programación por metas para la elaboración del contrato-programa de un hospital público. REDALYC, 73-88.

Louit, D. (Julio de 2012). Mantenimiento: Un proceso Estratégico. Seminario de Acercamiento Tecnológico. Calama, Chile.

Martínez S., I. A., Vertiz C., G., López P., J. F., Jiménez L., G., \& Moncayo M., L. A. (2014). Investigación de Operaciones. México: Grupo Editorial Patria.

Mora G., A. (2013). Mantenimiento. Planeación, ejecución y control. México: Alfaomega.

Moreno Vázquez, P., \& Castillo Valdez, O. D. (2018). El Mantenimiento Productivo Total "TPM" como factor para el aumento de la productividad y el nivel de aceptación del producto terminado. Revista de Ingeniería Industrial Marzo 2018 Vol.2 No.3, 1-9.

Moubray, J. (2004). Mantenimiento Centrado en Confiabilidad. North Carolina: Aladon LLC.

Rey S., F. (2003). Mantenimiento total de la producción. Proceso de implantación y desarrollo. Madrid: Confemetal.

Romero, C. (1993). Teoría de la desición multicriterio: conceptos, técnicas y aplicaciones. Alianza Editorial.

Sadi. (9 de Septiembre de 2014). Grupo Gaherma: Mantenimiento correctivo, preventivoy predictivo. ¿Cuál es el porcentaje ideal? Obtenido de http://blog.gaherma.com/index.php/2014/09/09/ mantenimiento-correctivo-preventivo-ypredictivo-cual-es-el-porcentaje-ideal/

Taha, H. (2012). Investigación de operaciones. México: Pearson Educación.

Tavares, L. (s.f.). Administración moderna del mantenimiento. Brasil: Novo Polo Publicación. 